\title{
Quantization of adiabatic pumped charge in the presence of superconducting lead
}

\author{
Jian Wang and Baigeng Wang \\ Department of Physics, The University of Hong Kong, Pokfulam Road, Hong Kong, China
}

(Received 19 October 2001; published 3 April 2002)

\begin{abstract}
We investigate the parametric electron pumping of a double barrier structure in the presence of a superconducting lead. The parametric pumping is facilitated by cyclic variation of the barrier heights $x_{1}$ and $x_{2}$ of the barriers. In the weak-coupling regime, there exists a resonance line in the parameter space $\left(x_{1}, x_{2}\right)$ so that the energy of the quasibound state is in line with the incoming Fermi energy. Levinson et al. found recently that the pumped charge for each pumping cycle is quantized with $Q=2 e$ for normal structure when the pumping contour encircles the resonance line. In the presence of a superconducting lead, we find that the pumped charge is quantized with the value $2 e$.
\end{abstract}

DOI: 10.1103/PhysRevB.65.153311

PACS number(s): 73.23.Ad, 73.40.Gk, 72.10.Bg, 74.50.+r

Physics of parametric electron pump has attracted great attention recently. ${ }^{1-12}$ A classical example of electron pump is the Thouless pump facilitated by a traveling wave potential. $^{13}$ The pumped charge is quantized ${ }^{13}$ and can be used as a quantum standard for electric charge. ${ }^{14}$ The quantization of pumped charge has also been studied for a large, almost open quantum dot $\operatorname{dot}^{15,16}$ and a small, strongly pinched quantum dot. ${ }^{17}$ In the latter case, there exists a resonance line along which the transmission through the quantum dot is at resonance. The pumped charge is quantized if the pumping contour in parameter space is properly chosen to encircle the resonance line. ${ }^{17}$ Recently, we have studied the parametric pumping in presence of a superconducting lead. ${ }^{18}$ At the normal-conductor-superconductor (NS) interface, an incoming electronlike excitation can be Andreev reflected as a holelike excitation. ${ }^{19}$ In contrast to the current doubling effect, ${ }^{20}$ we found that due to the quantum interference of direct reflection and the multiple Andreev reflection, the pumped current is four times of the value when the leads are normal in the weak pumping regime. In this paper, we explore the effect of superconducting lead on electron pumping in the opposite limit, i.e., we study the pumped charge during the pumping cycle in the the strong pumping regime. Here the pumped charge is equal to the pumped current multiplied by the period of pumping cycle. Similar to the Ref. 17, we examine the behavior of pumped charge near the resonance line. We find that the pumped charge in one pumping cycle is quantized with the value of $Q=2 e$ when one of the leads is superconducting. ${ }^{21}$

We consider a parametric pump, which consists of a double barrier tunneling structure attached to a normal left lead and a superconducting right lead. Due to the cyclic variation of external parameters $x_{1}$ and $x_{2}$, the adiabatic charge transfer in the presence of a superconducting lead is $1,22,23$

$$
Q^{N S}=2 e \int_{0}^{\tau} d t\left[\frac{d N_{L}}{d x_{1}} \frac{d x_{1}}{d t}+\frac{d N_{L}}{d x_{2}} \frac{d x_{2}}{d t}\right],
$$

where $\tau$ is the period of cyclic variation and the quantity $d N_{L} / d x$ is the injectivity ${ }^{25,26}$ given, at zero temperature, by

$$
\frac{d N_{L}}{d x_{j}}=\frac{1}{2 \pi} \operatorname{Im}\left[\mathcal{S}_{e e}^{*} \frac{\partial \mathcal{S}_{e e}}{\partial x_{j}}-\mathcal{S}_{h e}^{*} \frac{\partial \mathcal{S}_{h e}}{\partial x_{j}}\right],
$$

where the first term is the injectivity of the electron due to the variation of the external parameter, ${ }^{25,26}$ i.e., the partial density of states for an electron coming from the left lead and exiting the system as an electron, and the second term is the injectivity of a hole, i.e., the DOS for a hole coming from the left lead and exiting the system as an electron. Using the Green's theorem, the pumped charge can be expressed as surface integral over area $A$ enclosed by the path $\left(x_{1}(t), x_{2}(t)\right)$ in the parameter space ${ }^{1}$

$$
Q^{N S}=\frac{2 e}{\pi} \int_{A} d x_{1} d x_{2} \Pi^{N S}\left(x_{1}, x_{2}\right)
$$

with

$$
\Pi^{N S}\left(x_{1}, x_{2}\right)=\operatorname{Im}\left[\frac{\partial \mathcal{S}_{e e}^{*}}{\partial x_{1}} \frac{\partial \mathcal{S}_{e e}}{\partial x_{2}}-\frac{\partial \mathcal{S}_{h e}^{*}}{\partial x_{1}} \frac{\partial \mathcal{S}_{h e}}{\partial x_{2}}\right] .
$$

Note that the area $A$ is a measure of variation of pumping parameters $x_{1}$ and $x_{2}$. A is very small in the weak pumping limit while it remains finite in the strong pumping regime.

For the NS structures, the scattering matrix is described by $2 \times 2$ matrix $\hat{\mathcal{S}}$ when the Fermi energy is within the superconducting gap $\Delta$

$$
\hat{\mathcal{S}}=\left(\begin{array}{ll}
\mathcal{S}_{e e} & \mathcal{S}_{e h} \\
\mathcal{S}_{h e} & \mathcal{S}_{h h}
\end{array}\right),
$$

where $\mathcal{S}_{e e}\left(\right.$ or $\left.\mathcal{S}_{h e}\right)$ is the scattering amplitude of the incident electron reflected as an electron (or a hole). Using Andreev approximation, ${ }^{19}$ we have $\mathrm{e}^{20,27}$

$$
\hat{\mathcal{S}}=\hat{S}_{11}+\hat{S}_{12}\left(1-\hat{R}_{I} \hat{S}_{22}\right)^{-1} \hat{R}_{I} \hat{S}_{21}
$$

where $\hat{S}_{\beta \gamma}(E)(\beta, \gamma=1,2)$ is a diagonal $2 \times 2$ scattering matrix for the double barrier structure with matrix element $S_{\beta \gamma}(E)$ and $S_{\beta \gamma}^{*}(-E)$. For instance, we have

$$
\hat{S}_{11}=\left(\begin{array}{ll}
S_{11}(E) & 0 \\
0 & S_{11}^{*}(-E)
\end{array}\right) .
$$


In Eq. (6) $\hat{R}_{I}=\alpha \sigma_{x}$ is the $2 \times 2$ scattering matrix at NS interface due to the Andreev reflection with off-diagonal matrix element $\alpha$. Here $\alpha=\left(E-i \nu \sqrt{\Delta^{2}-E^{2}}\right) / \Delta$ with $\nu=1$ when $E>-\Delta$ and $\nu=-1$ when $E<-\Delta$. In Eq. (6), the energy $E$ is measured relative to the chemical potential $\mu$ of the superconducting lead. Equation (6) has a clear physical meaning. ${ }^{27}$ The first term is the direct reflection from the normal scattering structure and the second term can be expanded as $\hat{S}_{12} \hat{R}_{I} \hat{S}_{21}+\hat{S}_{12} \hat{R}_{I} \hat{S}_{22} \hat{R}_{I} \hat{S}_{21}+\cdots$, which is clearly the sum of the multiple Andreev reflection in the hybrid structure. It is the quantum interference of these two terms which gives rise the enhancement of pumped current in the weak pumping regime for NS system. ${ }^{18}$ From Eq. (6) we obtain the well-known expressions for the scattering matrix $\mathcal{S}_{e e}$ and $\mathcal{S}_{h e}$ (Ref. 20)

$$
\begin{aligned}
\mathcal{S}_{e e}(E)= & S_{11}(E)+\alpha^{2} S_{12}(E) S_{22}^{*}(-E) M_{e} S_{21}(E), \\
& \mathcal{S}_{h e}(E)=\alpha S_{12}^{*}(-E) M_{e} S_{21}(E),
\end{aligned}
$$

and

$$
M_{e}=\left[1-\alpha^{2} S_{22}(E) S_{22}^{*}(-E)\right]^{-1} .
$$

The double barrier structure, which we consider, is modeled by potential $U(y)=V_{1} \delta(y+a / 2)+V_{2} \delta(y-a / 2)$, where $V_{1}$ and $V_{2}$ are barrier heights that vary in a cyclic fashion to allow the charge pumping. For this system the retarded Green's function $G^{r}\left(y, y^{\prime}\right)$ can be calculated exactly. ${ }^{30}$ This is done by applying the Dyson's equation regarding the fact that any one of the $\delta$ barrier is just a perturbation of the remaining system. This way $G^{r}\left(y, y^{\prime}\right)$ is obtained by applying Dyson's equation twice starting from the Green's function of the one-dimensional free space. With $G^{r}\left(y, y^{\prime}\right)$ we can calculate scattering matrix exactly from the Fisher-Lee relation $^{28}$

$$
S_{\beta \gamma}=-\delta_{\beta \gamma}+i v G_{\beta \gamma}^{r},
$$

where $G_{\beta \gamma}^{r}=G^{r}\left(y_{\beta}, y_{\gamma}\right)$ and $v=2 k$ is the electron velocity in the normal lead. For normal structure, we have ${ }^{17}$

$$
\begin{aligned}
& S_{11}=\left[1-i x_{2}-\left(1+i x_{1}\right) \sigma^{2}\right] / D, \\
& S_{22}=\left[1-i x_{1}-\left(1+i x_{2}\right) \sigma^{2}\right] / D,
\end{aligned}
$$

and

$$
S_{12}=S_{21}=x_{1} x_{2} \sigma / D,
$$

where $D=-\left(1-i x_{1}\right)\left(1-i x_{2}\right)+\sigma^{2}, x_{1,2}=2 k V_{1,2}$, and $\sigma$ $=\exp (i k a)$. For the double barrier structure, the resonant tunneling is mediated by the quasibound state. When the energy of the incident electron is in line with the energy of the quasibound state the transmission coefficient reaches its maximum. The energy of quasibound states can be determined either by looking at the pole of the scattering matrix, ${ }^{17}$ which works well in one dimension, or by calculating the dwell time of the incident electron for two- or threedimensional systems. ${ }^{29}$ In the case of double $\delta$ barriers structure, the energy of quasibound state is given by ${ }^{17} E=E_{r}$ $+\Delta E$ with $\Delta E=-\left(k_{r} / a\right)\left(x_{1}+x_{2}\right)$, where $E_{r}=k_{r}^{2}$ $=(n \pi / a)^{2}$ is the energy of the bound state when the system is isolated. This defines a resonance line $x_{1}+x_{2}=-\delta$ in parameter space $\left(x_{1}, x_{2}\right)$ along which the transmission is at resonance. ${ }^{17}$ Here $\delta<0$ is the detuning of the Fermi energy from the bound state.

To show the quantization of charge transfer in the NS system, it is useful to recall the calculation of the normal case and make the comparison. In the normal case the charge transfer is given by ${ }^{1,21}$

$$
\begin{gathered}
Q^{N}=\frac{2 e}{\pi} \int_{A} d x_{1} d x_{2} \Pi^{N}\left(x_{1}, x_{2}\right), \\
\Pi^{N}\left(x_{1}, x_{2}\right)=\operatorname{Im}\left[\frac{\partial \mathcal{S}_{11}^{*}}{\partial x_{1}} \frac{\partial \mathcal{S}_{11}}{\partial x_{2}}+\frac{\partial \mathcal{S}_{12}^{*}}{\partial x_{1}} \frac{\partial \mathcal{S}_{12}}{\partial x_{2}}\right] .
\end{gathered}
$$

The pumped charge in this case has been calculated in Ref. 17. In the weak pumping limit, it is easy to show that only $\partial_{x} S_{11}$ contributes to the pumped charge. In the strong pumping regime, we will show in the following that the contribution from $\partial_{x} S_{12}$ to the pumped charge in normal structure is zero. As discussed in detail in Ref. 17, we neglect the smooth energy dependence of $x_{1}$ and $x_{2}$. From Eq. (16), we obtain the contribution due to $\partial_{x} S_{12}$

$$
\Pi_{1}^{N}\left(x_{1}, x_{2}\right)=F_{1}\left(x_{1}, x_{2}\right) / F_{2}^{2}\left(x_{1}, x_{2}\right)
$$

with

$$
\begin{gathered}
F_{1}\left(x_{1}, x_{2}\right)=-2 x_{1} x_{2}\left(x_{1}-x_{2}\right) \sin ^{2}(\delta / 2), \\
F_{2}\left(x_{1}, x_{2}\right)=x_{1}^{2} x_{2}^{2}+\left(x_{1}+x_{2}\right)^{2}+2\left(x_{1}+x_{2}\right) \sin \delta \\
+2\left(1-x_{1} x_{2}\right)(1-\cos \delta) .
\end{gathered}
$$

To compute the surface integral of $\Pi_{1}^{N}$ in Eq. (17), it is convenient to change the variables from $x_{1,2}$ to $p$ and $z$,

$$
x_{1}=-p \delta(1+z) / 2
$$

and

$$
x_{2}=-p \delta(1-z) / 2
$$

with $0<p<\infty$ and $-1<z<1$. Substituting Eqs. (20) and (21) into Eqs. (18) and (19) and expanding Eqs. (18) and (19) in terms of small $\delta$, we have

$$
\begin{gathered}
F_{1}=z\left(1-z^{2}\right) \delta^{5} p^{3} / 8 \\
F_{2}=\delta^{2}\left[(1-p)^{2}+\delta^{2} g(p, z)\right]
\end{gathered}
$$

where $g(p, z)$ [an even function of $z$ ] is given in Eq. (8) of Ref. 17. Since $F_{1}$ is an odd function of $z$, the contribution due to $\partial_{x} S_{12}$ to the pumped charge is zero.

Now we follow the same procedure to calculate the pumped charge for the NS system. For the parametric pumping at zero temperature, we only need the scattering matrix at the Fermi level, i.e., at $E=0$. From Eqs. (9) and (10), we see 

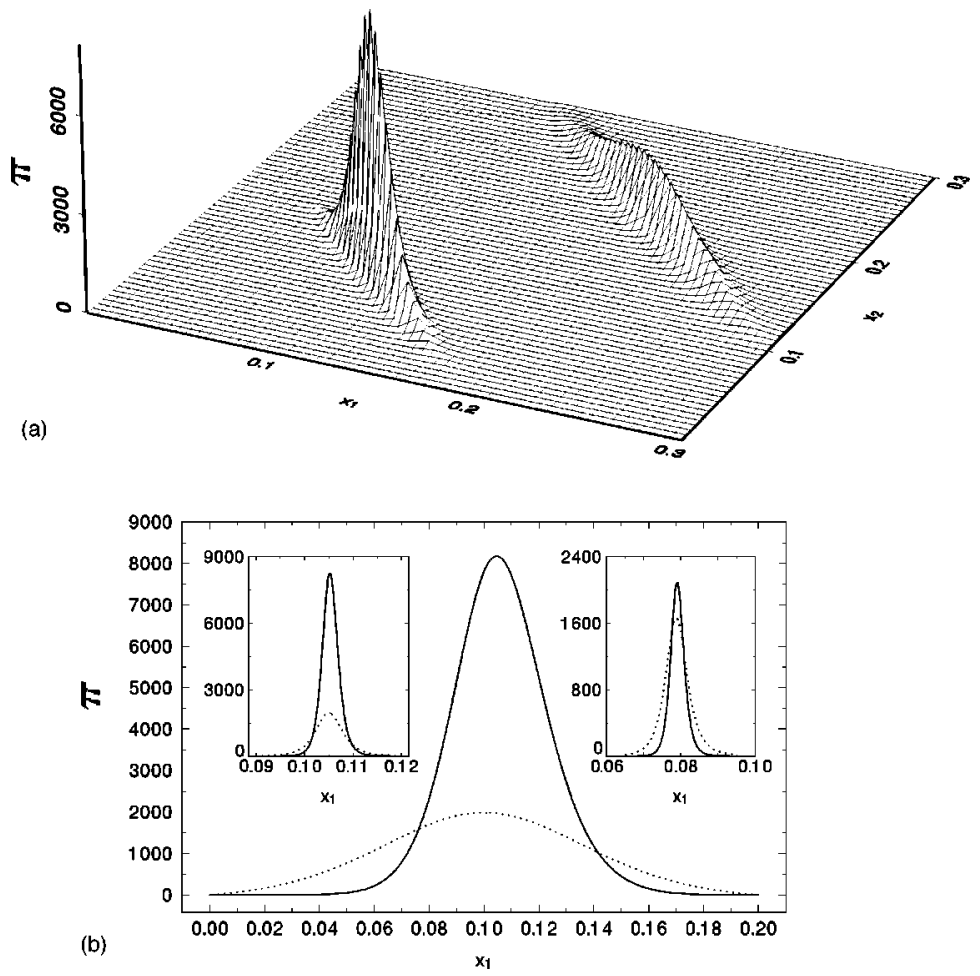

that $\mathcal{S}_{h e}$ is a real quantity and hence makes no contribution to the pumped charge in Eq. (3). It is straightforward to calculate $\Pi^{N S}$ using Eq. (8), from which we obtain, $\Pi^{N S}\left(x_{1}, x_{2}\right)=F_{3}\left(x_{1}, x_{2}\right) / F_{4}^{3}\left(x_{1}, x_{2}\right)$, where $F_{3}=4 x_{1}^{4} x_{2}^{3}(2$ $\left.-2 \cos \delta+x_{2} \sin \delta\right)$ and $F_{4}=x_{1}^{2} x_{2}^{2}+2\left(x_{1}+x_{2}\right)^{2}+4\left(x_{1}\right.$ $\left.+x_{2}\right) \sin \delta+4\left(1-x_{1} x_{2}\right)(1-\cos \delta)$.

In Fig. 1 we plot both $\Pi^{N S}$ and $\Pi^{N}$ as well as their cross sections along and perpendicular to the resonance line. We see that $\Pi^{N S}$ and $\Pi^{N}$ are peaked around the resonance line. Two features are worth noticing. First of all, the peak of $\Pi^{N S}$ is much sharper than that of $\Pi^{N}$. This is understandable and is due to the resonance nature of NS structures near the resonance line. In the Breit-Wigner form, the transmission coefficients for normal and NS structures are, respectively, $\left|S_{21}\right|^{2}=\Gamma_{1} \Gamma_{2} /\left[\left(E-E_{r}\right)^{2}+\Gamma^{2} / 4\right]$ and (see Ref. 20) $\left|\mathcal{S}_{h e}\right|^{2}$ $=4 \Gamma_{1}^{2} \Gamma_{2}^{2} /\left[4\left(E-E_{r}\right)^{2}+\Gamma_{1}^{2}+\Gamma_{2}^{2}\right]^{2}$, where $E_{r}$ is the resonant level, $\Gamma_{1}$ and $\Gamma_{2}$ are the decay widths into the left and right leads. Hence $\left|\mathcal{S}_{h e}\right|^{2}$ decays much faster away from $E_{r}$ than $\left|S_{21}\right|^{2}$. The scattering matrix $S_{21}$ and $\mathcal{S}_{h e}$ will appear, respectively, in Eqs. (3) and (15) implicitly as can be seen from Fisher-Lee relation Eq. (11) and the Dyson equation $\partial_{X_{2}} G_{11}^{r}$ $=G_{12}^{r} G_{21}^{r} \cdot{ }^{31}$ Second, the peak height of $\Pi^{N S}$ is four times larger than that of $\Pi^{N}$. This is precisely due to the constructive interference of direct reflection and multiple Andreev reflection. ${ }^{18}$ Now the physics of pumping at resonance is clear. For the resonance pumping in the weak pumping regime, we are looking at the small neighborhood of the peak. The area of the neighborhood has to be small since it is the weak pumping. The neighborhood has to be around the peak with $x_{1} \sim x_{2}$, since only around the peak the transmission coefficient is approximately 1 . As a result, we obtain immediately that the pumped charge or pumped current of the NS structure near the resonance is four times that of the corre-
FIG. 1. (a). The integrand $\Pi$ of Eqs. (3) and (15) as a function of $x_{1}$ and $x_{2}$ for $\delta=-0.2$. For illustration, the origin of $\Pi^{N}\left(x_{1}, x_{2}\right)$ has been shifted by $(0.1,0.1)$. (b). The cross section of $\Pi$ along the resonance line $x_{1}+x_{2}=-\delta$. Solid line, $\Pi^{N S}$; dotted line, $\Pi^{N}$. Insets show the cross section of $\Pi$ along the direction $x_{1}-x_{2}$ $=c_{0}$, which is perpendicular to the resonance line. Left inset, $c_{0}=0.01$; right inset, $c_{0}$ $=-0.042$. sponding normal structure. In the other extreme, for strong pumping, we take a large contour enclosing entire resonance line. Since $\Pi^{N S}$ decreases much faster than $\Pi^{N}$ away from the peak, it is understandable that the pumped charges (the integral of $\Pi$ over the area enclosed by the contour) for both normal and NS structures are equal, which will be shown analytically below.

After the expansion in powers of $\delta$ in Eqs. (18) and (19) and keeping the leading orders of $\delta$, we have

$$
\begin{aligned}
F_{3}= & p^{7}[2+p(-1+z)](-1+z)^{3}(1+z)^{4} \frac{\delta^{9}}{64}, \\
F_{4}= & 2(1-p)^{2} \delta^{2}+\left[-\frac{1}{6}+\frac{2 p}{3}+\frac{1}{2} p^{2}\left(-1+z^{2}\right)\right. \\
& \left.+\frac{1}{16} p^{4}\left(-1+z^{2}\right)^{2}\right] \delta^{4} .
\end{aligned}
$$

So Eq. (3) becomes

$$
Q^{N S}=\frac{e}{\pi} \int_{0}^{\infty} p d p \int_{-1}^{1} d z \frac{F_{3}}{F_{4}^{3}} \delta^{2},
$$

using the fact that $\lim _{\delta->0} \delta^{5} /\left(x^{2}+\delta^{2}\right)^{3}=(3 / 8) \pi \delta(x)$, Eq. (26) becomes

$$
Q^{N S}=3 \sqrt{2} e \int_{-1}^{1} d z \frac{\left(1-z^{2}\right)^{3}(1+z)^{2}}{\left(1+6 z^{2}+z^{4}\right)^{5 / 2}}=2 e .
$$

Hence the pumped charge for NS system is quantized at the same value as that of the normal structure.

Now we have a better physical picture for the transport properties of the NS structure. For the conductance or the 
$I$-V curve, we need $S_{21}$ or $\mathcal{S}_{h e}$. For normal structure, the current is given by $I^{N}=2 e / h \int d E\left[f\left(E-e V_{1}\right)-f(E\right.$ $\left.\left.-e V_{2}\right)\right]\left|S_{12}\right|^{2}$ and hence at resonance and at zero temperature $G^{N}=I^{N} /\left(V_{1}-V_{2}\right)=2 e^{2} / h$. For NS structure, we have $^{20,32} \quad I^{N S}=2 e / h \int d E\left[f\left(E-e V_{1}\right)-f\left(E+e V_{1}\right)\right]\left|\mathcal{S}_{h e}\right|^{2}$ and at resonance $G^{N S}=I^{N S} / V_{1}=4 e^{2} / h$, which is the wellknown doubling of the conductance. For pumped charge or pumped current at resonance, however, it depends only on $\partial_{x_{i}} S_{11}$ or $\partial_{x_{i}} \mathcal{S}_{e e}(E=0$ is assumed). Due to the constructive interference between direct reflection and multiple Andreev reflection in the weak pumping regime, the charge transfer increases by a factor of 4 when one of the leads becomes superconducting. In the strong pumping regime, however, the charge transfer is quantized at the value equal to that of normal structure, if the pumping contour is chosen such that the resonance line is enclosed. The physics behind this can be understood as follows. In the normal case, the contour enclosing the resonance line in the parameter space passes through the resonance line at two points $\left(x_{1}, x_{2}\right)=(0,-\delta)$ when the left contact is almost closed and $(-\delta, 0)$ when the right contact is almost closed. When passing through these two points, the resonance level of the dot crosses the Fermi energy. At each crossing, the occupation of the level changes, and two electrons with opposite spins enter or exit the region between the barriers. Since one of the tunnel barriers has zero conductance at these points, it is clear that the electrons must have tunneled through the other contact upon entering or leaving the quantum dot. Hence, in the pumping cycle, electrons are shuttled pairwise through the dot. In the presence of the superconducting lead, the resonance level (both the energy and the width) is exactly the same as that of normal case since the scattering matrix is given by $\mathcal{S}_{\text {he }}$ $=i\left|S_{12}\right|^{2} /\left(1+\left|S_{22}\right|^{2}\right)$ when $E=0$. Therefore, the same argument applies to the superconducting case and the quantization unit is $2 e$. Note that our statement is only valid when the electron interaction is neglected. For the case of two normalmetal contacts, if interactions are included the quantization will remain, but now the quantum is only $e$ : Only one electron at a time can enter the region between the barriers; addition of a second electron is forbidden by the Coulomb blockade. In the presence of the superconducting lead, since the Andreev reflection requires two electrons with opposite spins in order to produce the supercurrent, it seems that the pumping is not allowed in the strong pumping regime due to the Coulomb blockade. In this paper, we have also neglected effects of the temperature and inelastic scattering. As discussed in Ref. 17 the temperature will destroy the quantization of the pumped charge. When inelastic channel is present an additional physical mechanism for an incoherent pump effect will show up. ${ }^{33}$

We gratefully acknowledge support by a RGC grant from the SAR Government of Hong Kong under Grant No. HKU 7215/99P.
${ }^{1}$ P.W. Brouwer, Phys. Rev. B 58, R10 135 (1998).

${ }^{2}$ M. Switkes et al., Science 283, 1905 (1999).

${ }^{3}$ F. Zhou, B. Spivak, and B.L. Altshuler, Phys. Rev. Lett. 82, 608 (1999)

${ }^{4}$ M. Wagner, Phys. Rev. Lett. 85, 174 (2000).

${ }^{5}$ J.E. Avron et al., Phys. Rev. B 62, R10 618 (2000).

${ }^{6}$ I.L. Aleiner, B.L. Altshuler, and A. Kamenev, Phys. Rev. B 62, 10373 (2000).

${ }^{7}$ Y.D. Wei, J. Wang, and H. Guo, Phys. Rev. B 62, 9947 (2000).

${ }^{8}$ M.G. Vavilov, V. Ambegaokar, and I.L. Aleiner, Phys. Rev. B 63, 195313 (2001)

${ }^{9}$ P.W. Brouwer, Phys. Rev. B 63, 121303 (2001); M.L. Polianski and P.W. Brouwer, ibid. 64, 075304 (2001).

${ }^{10}$ P. Sharma and C. Chamon, Phys. Rev. Lett. 87, 096401 (2001).

${ }^{11}$ X.B. Wang and V.E. Kravtsov, Phys. Rev. B 64, 033313 (2001).

${ }^{12}$ Y.D. Wei et al., Phys. Rev. B 64, 115321 (2001).

${ }^{13}$ D.J. Thouless, Phys. Rev. B 27, 6083 (1983).

${ }^{14}$ Q. Niu, Phys. Rev. Lett. 64, 1812 (1990).

${ }^{15}$ I.L. Aleiner and A.V. Andreev, Phys. Rev. Lett. 81, 1286 (1998).

${ }^{16}$ T.A. Shutenko, I.L. Aleiner, and B.L. Altshuler, Phys. Rev. B 61, 10366 (2000).

${ }^{17}$ Y. Levinson, O. Entin-Wohlman, and P. Wolfle, Physica A 302, 335 (2001).

${ }^{18}$ J. Wang et al., Appl. Phys. Lett. 79, 3977 (2001).
${ }^{19}$ A.F. Andreev, Zh. Éksp. Teor. Fiz. 46, 1823 (1964) [Sov. Phys. JETP 19, 1228 (1964)].

${ }^{20}$ C.W.J. Beenakker, Rev. Mod. Phys. 69, 731 (1997).

${ }^{21} \mathrm{~A}$ factor of 2 has been included for spin.

${ }^{22}$ The units are fixed by setting $\hbar=2 m=1$ in the following analysis. For the GaAs system with $a=1000 \AA$, the energy uint is $E=56 \mu \mathrm{eV}$.

${ }^{23}$ This formula can be derived using the Keldysh nonequilibrium Green's function method (see Ref. 24) and we have assumed that the Coulomb blockade effect can be neglected.

${ }^{24}$ B.G. Wang, J. Wang, and H. Guo, Phys. Rev. B 65, 073306 (2002).

${ }^{25}$ T. Gramespacher and M. Buttiker, Phys. Rev. B 61, 8125 (2000).

${ }^{26}$ J. Wang et al., Phys. Rev. B 64, 104508 (2001).

${ }^{27}$ G.B. Lesovik, A.L. Fauchere, and G. Blatter, Phys. Rev. B 55, 3146 (1997).

${ }^{28}$ D.S. Fisher and P.A. Lee, Phys. Rev. B 23, 6851 (1981).

${ }^{29}$ J. Wang, Y.J. Wang, and H. Guo, Appl. Phys. Lett. 65, 1793 (1994).

${ }^{30}$ M.K. Yip, J. Wang, and H. Guo, Z. Phys. B: Condens. Matter 104, 463 (1997).

${ }^{31}$ V. Gasparian, T. Christen, and M. Buttiker, Phys. Rev. A 54, 4022 (1996).

${ }^{32}$ Q.F. Sun, J. Wang, and T.H. Lin, Phys. Rev. B 59, 3831 (1999).

${ }^{33}$ M. Moskalets and M. Buttiker, Phys. Rev. B 64, 201305 (2001). 